\title{
Persistence of respiratory syncytial virus (RSV) infection and development of RSV-specific IgG1 response in a guinea-pig model of acute bronchiolitis
}

\author{
A. Dakhama, T.Z. Vitalis, R.G. Hegele
}

\begin{abstract}
Persistence of respiratory syncytial virus (RSV) infection and development of RSV-specific IgGl response in a guinea-pig model of acute bronchiolitis. A. Dakhama, T.Z. Vitalis, R.G. Hegele. CERS Journals Ltd 1997.

ABSTRACT: Acute respiratory syncytial virus (RSV) bronchiolitis in children can result in sequelae of recurrent wheezing and asthma and production of RSV-specific immunoglobulin $\mathrm{E}$ ( $\operatorname{IgE}$ ), but the pathogenesis of these sequelae is poorly understood. Guinea-pigs experimentally inoculated with human RSV show histological evidence of acute bronchiolitis and chronic persistence of viral antigens and genome in the lungs; whether this persistence is due to infectious replicating virus, and whether infected animals develop RSV-specific immunoglobulin G1 (IgG1) (the main class of antibody involved in guinea-pig allergic responses) is unknown.

Guinea-pigs were inoculated intranasally with human RSV or with uninfected cell culture supernatant. At times ranging 1-60 days postinoculation, the viral titre in the lung was determined by immunoplaque assay (a method combining viral culture and immunocytochemistry). Serum titres of RSV-specific IgG1 antibodies were determined by enzyme-linked immunosorbent assay. Bronchiolar inflammation was assessed on coded lung sections, by using a semiquantitative, histological scoring system based on features of human acute bronchiolitis.

Infectious RSV was cultured from the lungs of infected animals on all study days, with maximal viral replication observed on Day 3. RSV-specific IgG1 antibodies were detected in all RSV-inoculated animals from Day 7 onward, with the highest antibody titre measured on Day 28. RSV-inoculated guinea-pigs had maximal bronchiolar inflammation on Day 7, and had significantly increased polymorphonuclear cell infiltrates on Days 28 and 60.

Respiratory syncytial virus chronically persists as infectious virus in the guineapig lung. Infected animals develop an anti-respiratory syncytial virus immunoglobulin G1 antibody response, histological evidence of acute bronchiolitis, and chronic airway inflammation. Persistent respiratory syncytial virus lung infection may be important in the pathogenesis of postbronchiolitis wheezing and asthma in children.
\end{abstract}

Eur Respir J., 1997; 10: 20-26.

Pulmonary Research Laboratory, University of British Columbia, St. Paul's Hospital, Vancouver, BC, Canada.

Correspondence: R.G. Hegele Pulmonary Research Laboratory University of British Columbia St. Paul's Hospital Vancouver British Columbia

Canada

Keywords: Bronchiolitis guinea pig

persistent infection respiratory syncytial virus specific IgG1

Received: April 291996 Accepted after revision August 251996

This study was supported by the Medical Research Council of Canada (Operating Grant No. MT-12713), and the Respiratory Health Network of Centres of Excellence
Respiratory syncytial virus (RSV) is a major cause of acute bronchiolitis in infants and young children [1,2], and is associated with significant morbidity and mortality in seriously affected children [3]. Several epidemiological studies have suggested that occurrence of RSV bronchiolitis, during early life, constitutes an important risk factor for the development of subsequent asthma and allergy, probably in genetically predisposed children [4-7]. In children with documented RSV infection, increased titres of RSV-specific immunoglobulin E (IgE) antibody have been demonstrated in nasal secretions during and after RSV bronchiolitis [8,9], and have recently been demonstrated in the lower respiratory tracts during acute bronchiolitis [10]. However, the mechanisms by which RSV may be involved in the development of postbronchiolitis asthma and allergy are poorly understood.

Various animal models have been developed to investigate in vivo, in a time course manner, the histological, physiological and immunological responses that occur in the lung following RSV infection [11-13]. Most animal studies have focused on the acute or the convalescent phases of the disease, and only minor attention has, so far, been paid to later stages, presumably because viral replication and airway inflammation apparently resolve after 10-14 days [14, 15]. Acute bronchiolitis has also been reproduced in guinea-pigs inoculated with moderate doses of human RSV [16], with the subsequent finding that both RSV genome and proteins persist in guinea-pig lung for at least 60 days postinfection [17]. However, the replication of RSV in the lung of this animal model is not yet documented, and it is still unclear whether the virus may persist in the guinea-pig lung as infectious virus or as residual portions of inactivated virus. Although the guinea-pig is known to mount a virusspecific humoral response after intranasal instillation of human RSV [11], it is unknown whether guinea-pigs produce RSV-specific immunoglobulin G1 (IgG1), the 
functional equivalent to mouse $\operatorname{IgE}$ [18]. In addition, the airway inflammation that develops in this animal model has not been followed over the interval from the inoculation procedure to 60 days postinoculation, long after the resolution of acute bronchiolitis.

The present study was carried out to determine: 1) whether human RSV replicates in the guinea-pig lung during acute bronchiolitis; 2) whether RSV may persist as infectious virus in the guinea-pig lung after acute bronchiolitis; 3) whether this animal model can develop an anti-RSV specific IgG1 antibody response following RSV infection; and 4) whether RSV-inoculated animals show a different time course of bronchiolar inflammation over 60 days, when compared to control animals that undergo inoculation with uninfected cell culture supernatant.

\section{Materials and methods}

\section{Virus preparation}

The Long strain of type A RSV was obtained from American Type Culture Collection (ATCC) (Rockville, MD, USA). The virus stock was prepared in HEp-2 (ATCC) cell monolayers grown at $37^{\circ} \mathrm{C}$ and $5 \% \mathrm{CO}_{2}$ enriched atmosphere in minimal essential medium (MEM) (GibcoBRL, Life Technologies Inc., Grand Island, NY, USA) supplemented with $2 \%$ heat-inactivated foetal bovine serum (FBS) (HyClone Laboratories Inc., Logan, UT, USA), $0.292 \mathrm{mg} \cdot \mathrm{ml}^{-1} \mathrm{~L}$-glutamine (GibcoBRL) and $50 \mu \mathrm{g} \cdot \mathrm{mL}^{-1}$ gentamicin (GibcoBRL). When more than $75 \%$ of cells were involved in formation of syncytia, the virus was harvested after disrupting the cells in the same culture medium by shaking with $3 \mathrm{~mm}$ autoclaved glass beads. The collected fluid was then centrifuged at $4^{\circ} \mathrm{C}$ for $10 \mathrm{~min}$ at $10,000 \times \mathrm{g}$ to remove cell debris, and the resulting cleared supernatant was kept frozen in aliquots at $-70^{\circ} \mathrm{C}$ as stock virus inoculum. Sham inoculum was prepared by the same procedure from uninfected HEp-2 cell lysate. The virus titre in the inoculum was determined by plaque assay on the same day as the inoculation of guinea-pigs.

\section{Animals and inoculation procedure}

A total of 52 female Cam Hartley guinea-pigs (Charles River laboratories, Montréal, QC, Canada), each weighing 300-350 g, was used in this study. Animals were acclimatized for 1 week before inoculation. Twenty eight animals were inoculated intranasally with 30,000 plaqueforming units (pfu) of RSV in a total volume of $200 \mu \mathrm{L}$, and kept separated from uninfected animals in a different room. Control animals consisted of 24 sham-inoculated guinea-pigs. All the animals were inoculated under light anaesthesia, performed via inhalation of 5\% halothane. Preliminary experiments using colloidal carbon indicated that this inoculum size was sufficient to reach the lower respiratory tract in this animal (fig. 1).

\section{Study design}

Animals were studied on Days 1, 3, 7, 14, 28 and 60 following the inoculation. There were four animals per study day in the control group. In the RSV group, four

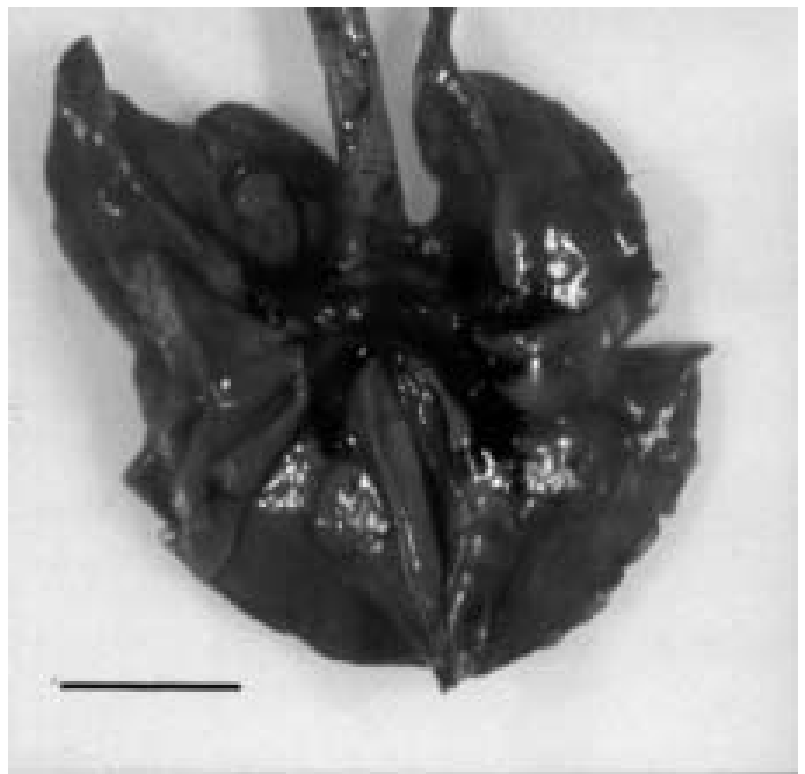

Fig. 1. - Macroscopic appearance of guinea-pig lung after intranasal inoculation with $200 \mu \mathrm{L}$ of colloidal carbon. Note the distribution of the liquid to large and small airways. Internal scale bar $=1 \mathrm{~cm}$.

infected animals were studied on each of Days 1, 3, 7 and 14, and six infected animals on each of days 28 and 60 . The numbers of animals studied at each time point were chosen based on results of previous studies of $\mathrm{RSV}$-infected guinea-pigs [16], to allow discrimination of virus-specific effects from nonspecific effects of the inoculation procedure. On each of Days 28 and 60, six RSV-infected animals were studied, based on previous observations of persistence of viral proteins and genome in the lungs of approximately $60-70 \%$ of infected animals [17].

\section{Blood collection and serum preparation}

At the designated time-points, guinea-pigs from each group were sacrificed by intraperitoneal injection of pentobarbital, and peripheral blood was collected by cardiac puncture using a $10 \mathrm{~mL}$ syringe equipped with $15-\mathrm{G}$ needle. Animal sera were separated from clotted blood at $4^{\circ} \mathrm{C}$ and kept frozen at $-70^{\circ} \mathrm{C}$ until needed.

\section{Determination of virus titre}

After dissection under sterile conditions, the right lung was weighed and kept frozen at $-70^{\circ} \mathrm{C}$ in a $15 \mathrm{~mL}$ tube containing $3 \mathrm{~mL}$ of MEM for viral culture. Replicating virus was detected in the lung by using a combined immunocytochemistry and plaque assay method, as follows: $0.5 \mathrm{~mL}$ aliquots of serial 10 fold dilutions of cleared supernatants prepared from lung tissue homogenates were incubated with monolayers of HEp-2 cells grown in sixwell culture plates. After $90 \mathrm{~min}$ of adsorption, the inoculum was aspirated off and the cells overlaid with $3 \mathrm{~mL}$ of $1 \%$ melted agarose-containing MEM supplemented with 5\% FBS. After solidification, the plates were incubated for culture until syncytia developed. The cells were then fixed for $2 \mathrm{~h}$ with $4 \%$ paraformaldehyde in phosphate-buffered saline (PBS) and, after removing the 
agarose overlay, the cells were washed three times for 5 min with $50 \mathrm{mM}$ Tris-buffered saline (TBS), pH 7.6. Syncytia were detected by immunocytochemical staining using a rabbit anti-RSV antibody (1:400) (Dako, Denmark) followed by biotin-conjugated goat anti-rabbit immunoglobulins (Dako) diluted 1:500 in TBS and alkaline phosphatase-conjugated avidin/biotin complex (Dako).

\section{Detection of RSV-specific IgG1 antibodies}

RSV-specific IgG1 antibodies were detected by enzyme-linked immunosorbent assay (ELISA), using a peroxidase-conjugated goat anti-guinea-pig IgG1 (Nordic Immunological Laboratories, Capistrano Beach, CA, USA). This antibody is specific to the Fc portion of the IgG1 molecule, and does not cross-react with other components of the immunoglobulin system or with other proteins present in the serum of guinea-pig. RSV antigens were prepared as described previously [19]. Polypropylene 96-well microtitre plates (Falcon) were precoated overnight at $4^{\circ} \mathrm{C}$ with $\mathrm{RSV}$ antigens $\left(2 \mu \mathrm{g}\right.$ proteins $\left.\cdot \mathrm{well}^{-1}\right)$ in coating buffer (Sigma Chemicals, St. Louis, MO, USA), and washed three times with PBS containing Tween-20 (PBST) $(10$ mM PBS, pH $7.4+0.05 \%$ Tween-20). After washing, $100 \mu \mathrm{L}$ of serially (twofold) diluted individual guinea-pig sera were added to each well for $2 \mathrm{~h}$ of incubation at room temperature, followed by three washes with PBST, addition of $200 \mu \mathrm{L}$ of peroxidase-conjugated goat anti-guinea-pig IgG1 (optimally diluted 1:2,000 in PBST) to each well and incubation for $2 \mathrm{~h}$ at room temperature. After three washes, $200 \mu \mathrm{L}$ of o-phenylenediamine dihydrochloride peroxidase substrate (Sigma) was added to each well for $30 \mathrm{~min}$ and the reaction was terminated by addition of $50 \mu \mathrm{L} 3 \mathrm{M} \mathrm{H}_{2} \mathrm{SO}_{4}$.

The optical density (OD) was determined at $492 \mathrm{~nm}$ in an ELISA microtitre plate reader (Titertek Multiskan Plus MK2). Titres were determined as the greatest serum dilution to produce an optical density at least $0.1 \mathrm{OD}$ units higher than control values. Controls consisted of wells precoated with uninfected HEp-2 cell proteins prepared following the same procedure as used for RSV antigens. The endpoint of $0.1 \mathrm{OD}$ units above control values was predetermined by point titration as the least amount of guinea-pig IgG1 that can be detected by the method using the same optimal dilution of anti-guineapig IgG1. This cut-off level was also selected based on a lower limit of 1.5-2 times background values.

\section{Histological assessment}

For each animal, the left lung was inflated with $5 \mathrm{~mL}$ of ornithine carbamyl transferase (OCT) (50\% in PBS) and cut in the sagittal plane into 3-5 mm thick, parallel slices. The lung tissue slices were then fixed at $4^{\circ} \mathrm{C}$ for $24 \mathrm{~h}$ with $4 \%$ paraformaldehyde in PBS, washed in PBS and processed into paraffin. Histological examination was performed on $5 \mu \mathrm{m}$ thick lung tissue sections stained with haematoxylin and eosin (H\&E), coded such that neither the RSV status (i.e. infected or control) nor the study day for a given slide was known to the microscopist.

Histopathological changes in the airways of all RSVinoculated and control guinea-pigs in this study were evaluated under light microscope by using a previously published, semiquantitative histological scoring method based on the features of human acute bronchiolitis, with minor modifications [16]. Briefly, 10 noncartilaginous, muscular airways per animal lung were evaluated for bronchiolar epithelial necrosis, mononuclear cell infiltrates and polymorphonuclear (PMN) cell infiltrates, by comparison to a panel of standardized photomicrographs. The observed score for each parameter was determined as the sum of individual airway scores ( 0 for normal, 2 for moderate-to-severe changes), such that the maximum score for each parameter was 20 per animal. Previous observations in this animal model have established that scoring these histological features permits distinction of viral effects on bronchiolar inflammation from nonspecific background [16].

\section{Statistical analysis}

Data are presented as mean \pm SD. For analysis of viral titres, data were log-transformed and analysed using a one-way analysis of variance (ANOVA). Histological scores between the RSV-inoculated and control groups over the 60 days of the study were analysed using a two-way ANOVA. A sequential rejective Bonferroni procedure was used to correct for multiple comparisons [20]. A p-value of $<0.05$ was considered significant.

\section{Results}

No apparent clinical symptoms were observed in guineapigs following infection with RSV, and no significant change in body weight gain was noted in comparison to the control group during the study period (fig. 2). Infectious RSV was isolated from the lung of all infected guinea-pigs, with the exception of one animal at Day 60 . Figure 3 shows a representative syncytium of HEp2 cells, as detected by immunostaining with a polyclonal anti-RSV antibody, after culture with lung tissue

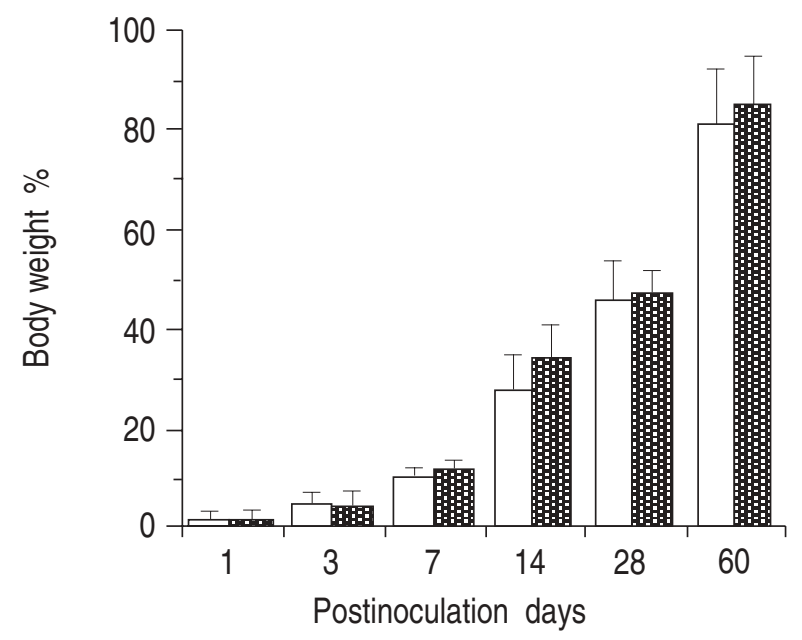

Fig. 2. - Body weight gain during the study period, calculated, as percentage of initial body weight, as follows: (final weight - initial weight) $\times 100 /$ initial weight. Values are presented as mean \pm sD. No statistically significant differences were observed between the two groups. $\square$ : sham; 


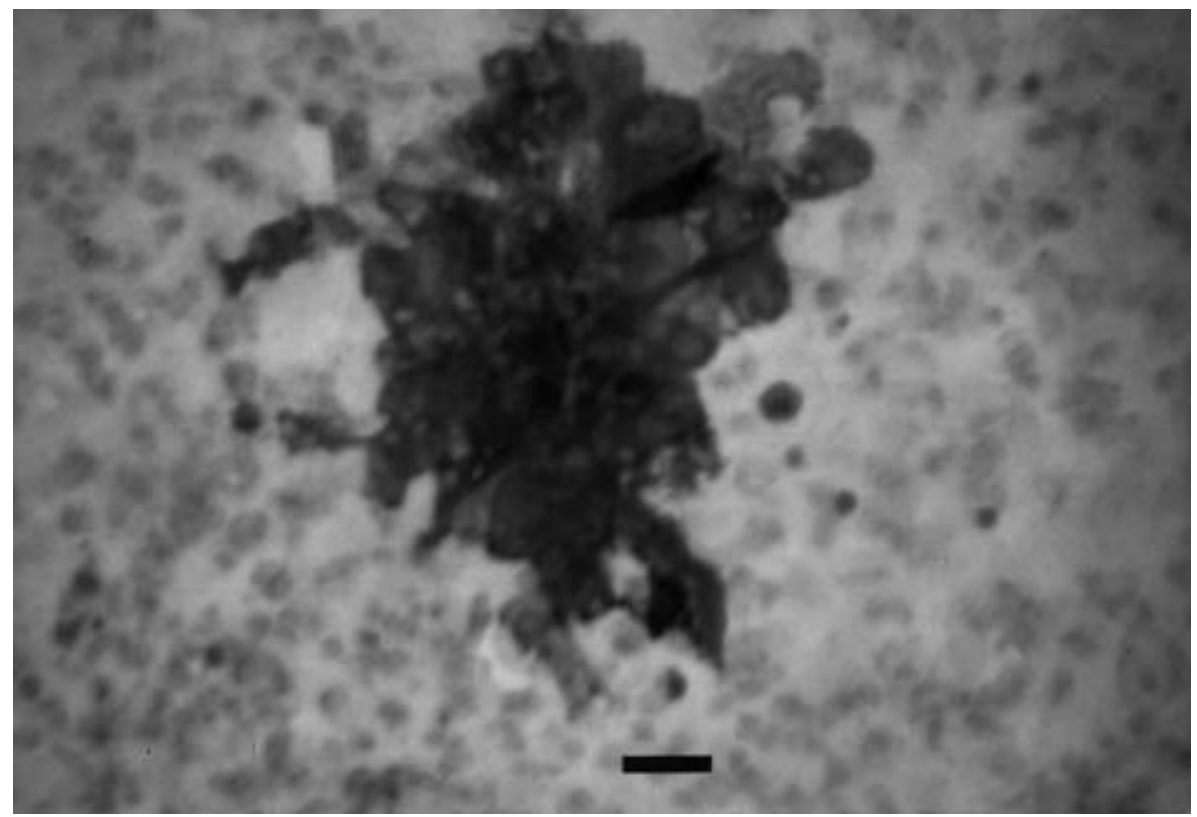

Fig. 3. - Typical appearance of respiratory synctial virus (RSV)-positive synctium of HEp-2 cells, as detected by immunohistocytochemistry, after 4-5 days of culture following addition of lung homogenates from RSV-infected guinea-pigs (Day 3). (Internal scale bar $=50 \mu \mathrm{m})$.

homogenates from RSV-infected guinea-pigs. As shown in figure 4 , the mean \pm SD titre of intrapulmonary replicating RSV was significantly increased from $577 \pm 267$ pfu. $\mathrm{g}^{-1}$ lung at Day 1, to a peak value of 3,936 $\pm 2,154$ $\mathrm{pfu} \cdot \mathrm{g}^{-1}$ at Day $3(\mathrm{p}<0.005)$. The viral titre gradually decreased, thereafter, to low levels of replication (18 \pm 17 pfu.g-1 at Day 28), which were maintained up to 60 days postinfection $\left(15 \pm 17 \mathrm{pfu} \cdot \mathrm{g}^{-1}\right)$. No virus was detected in the lungs of control guinea-pigs at any time during the study.

Figure 5 illustrates the reciprocal titres of anti-RSV IgG1 antibodies as detected by ELISA in the serum of infected guinea-pigs. No RSV-specific IgG1 antibodies were detected in the sera from control guinea-pigs. AntiRSV IgG1 antibodies were first detected on Day 7, and

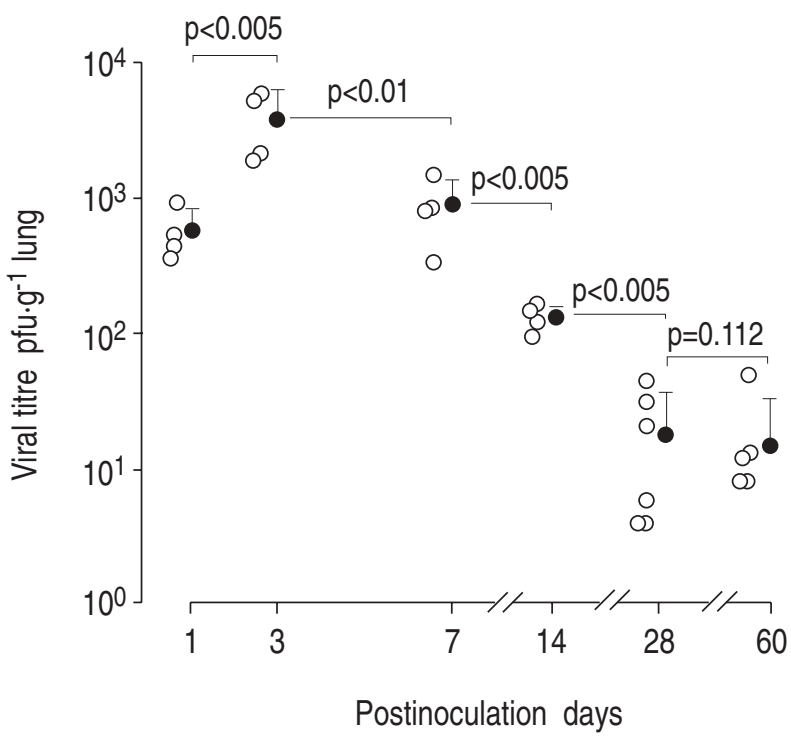

Fig. 4. - Titres of intrapulmonary replicating respiratory syncytial virus (RSV) recovered from guinea-pig lungs at various time-points following inoculation with RSV ( $O$ : individual data; $\bullet$ : mean $\pm \mathrm{SD}$ ). RSV was not isolated by culture in one animal on Day 60. The virus was not detected in the lungs of sham-inoculated guinea-pigs at any time of the study. pfu: plaque-forming units. all infected guinea-pigs developed this antibody. The mean \pm SD reciprocal titre of this serum antibody was significantly increased at Day $14(\mathrm{p}<0.05)$, and reached a peak level $(1,000 \pm 490)$ at Day 28 , before declining on Day 60 to levels comparable to those observed 7 days after infection.

Figure 6 shows representative photomicrographs of lung sections from RSV-infected guinea-pigs, 7 days post-inoculation, that demonstrate histological evidence of acute bronchiolitis. Figure 7 shows the results of histological scoring for bronchiolar inflammation in RSVinoculated and control guinea-pigs over 60 days. In comparison to control animals, the RSV group had significantly higher mean scores for bronchiolar epithelial

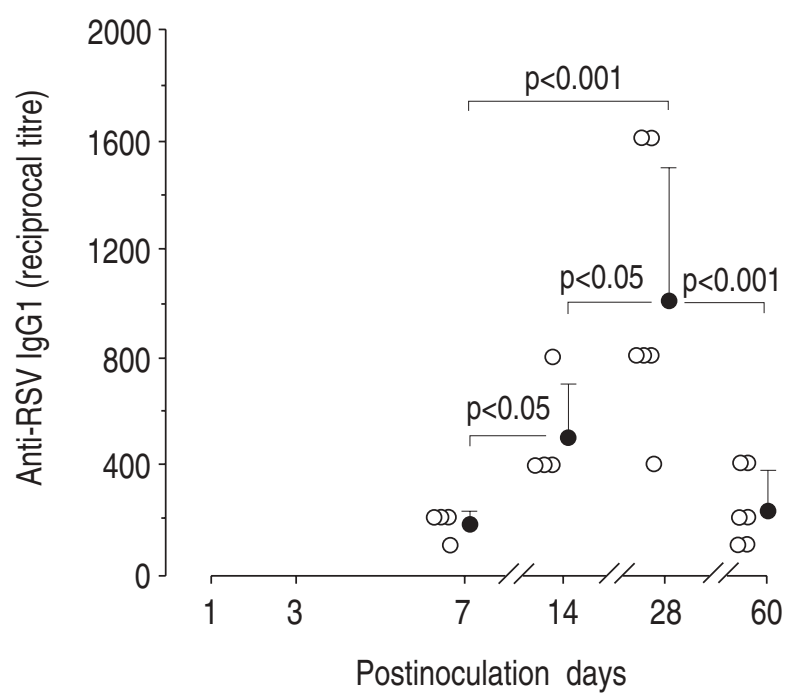

Fig. 5. - Titres of circulating respiratory syncytial virus (RSV)-specific IgG1 antibody (O: individual data; $\bullet$ : mean $\pm \mathrm{SD}$ ), as detected by ELISA in the sera from guinea-pigs at various time-points following inoculation with RSV. Anti-RSV IgG1 antibody was not detected in the sera from sham-inoculated guinea-pigs. ELISA: enzyme-linked immunosorbent assay; IgG1: immunoglobulin G1. 
a)

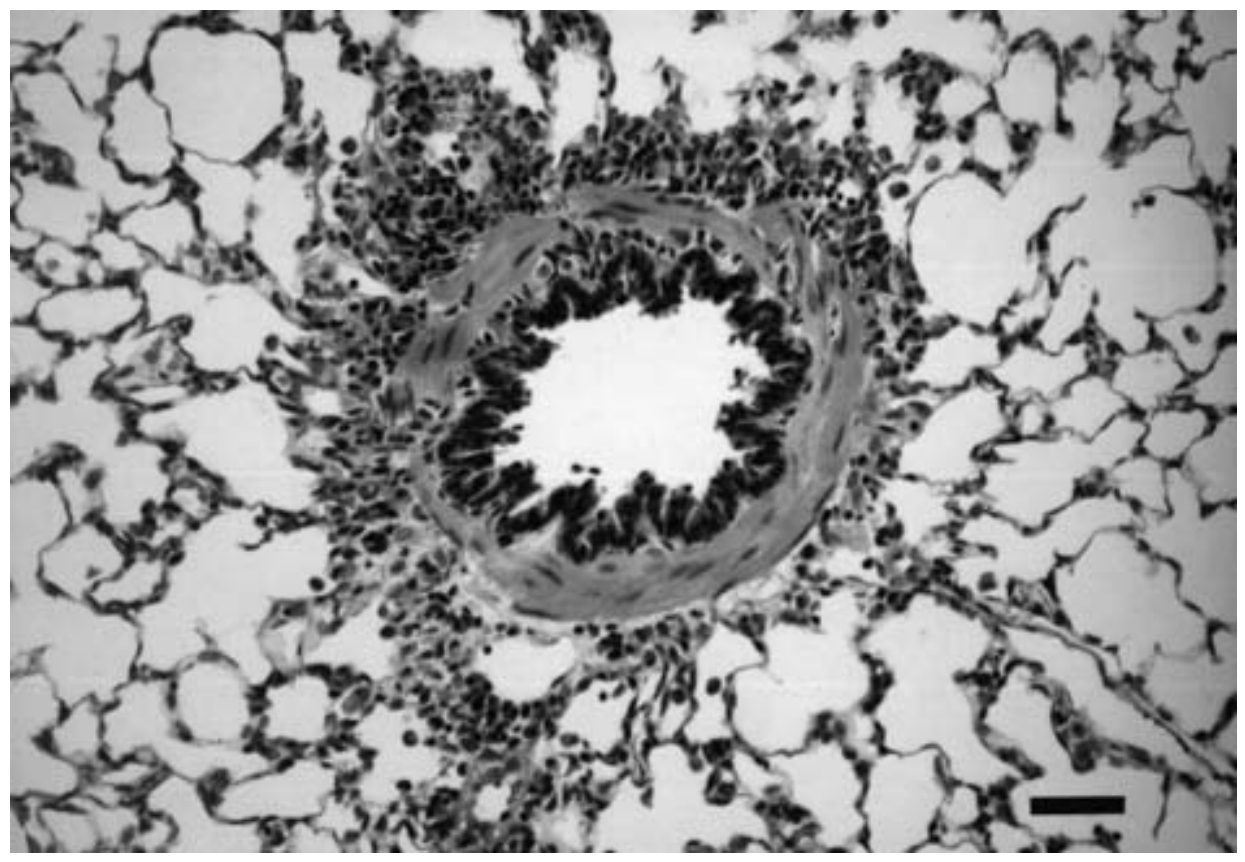

b)

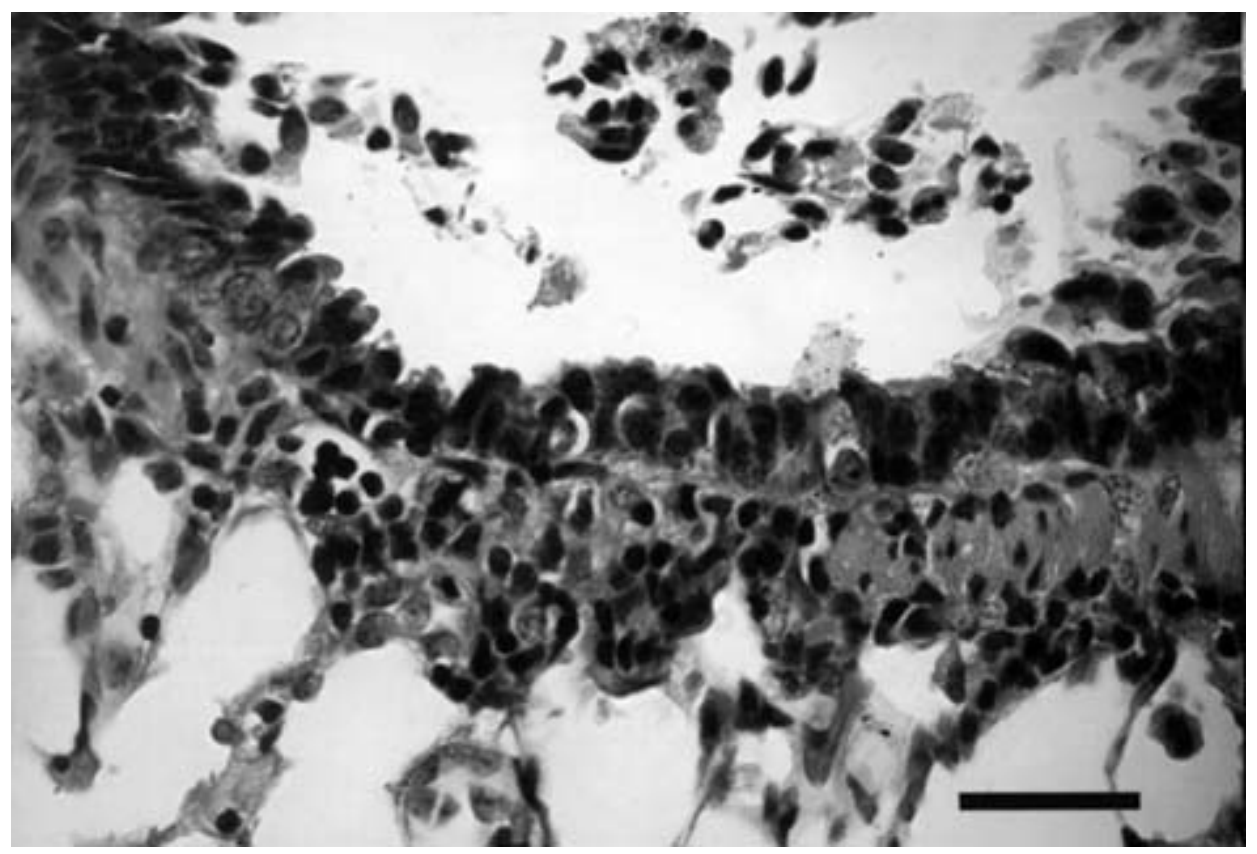

Fig. 6. - Photomicrographs of respiratory syncytial virus (RSV)-infected guinea-pig lung at Day 7 postinoculation. a) Membranous bronchiole with extensive peribronchiolar inflammatory infiltrates consisting of an admixture of mononuclear and polymorphonuclear leucocytes. b) Necrotic epithelial cells, and inflammatory cells with the airway lumen. Haematoxylin staining; internal scale bar $=50 \mu \mathrm{m}$.

necrosis (fig. 7a) and mononuclear cell infiltrates (fig. $7 b)$ during acute infection on day $7(\mathrm{p}<0.001)$. In addition, the RSV group had significantly higher mean scores for bronchiolar PMN infiltrates (fig. 7c) during acute infection on Days 3 and 7, and during persistent infection on Days 28 and $60(\mathrm{p}<0.009)$.

Within the RSV-inoculated group, analysis of histological scoring over 60 days revealed significantly higher mean scores for bronchiolar epithelial necrosis during acute lytic infection on Days 3 and 7 , when compared to the other study days $(\mathrm{p}<0.002)$. In addition, the mean score for mononuclear cell infiltrates was significantly higher on Day 7, in comparison to the other study days $(\mathrm{p}<0.001)$. Bronchiolar PMN infiltrates showed a biphasic pattern, with significant increases during acute infection that peaked on Day $7(\mathrm{p}<0.004$ in comparison to the other study days), followed by a significant decrease to control levels during convalescence on Day 14 ( $\mathrm{p}<$ 0.0002 compared to Day 7), and a subsequent increase that was sustained during persistent infection on Days 28 and $60(\mathrm{p}<0.003$ compared to Day 14). Within the control group, there were no significant differences in the mean scores for epithelial necrosis and mononuclear cell infiltrates over 60 days; however, a transient, significant increase in PMN infiltrates $(\mathrm{p}<0.02)$ was observed on Day 1 after sham inoculation. 


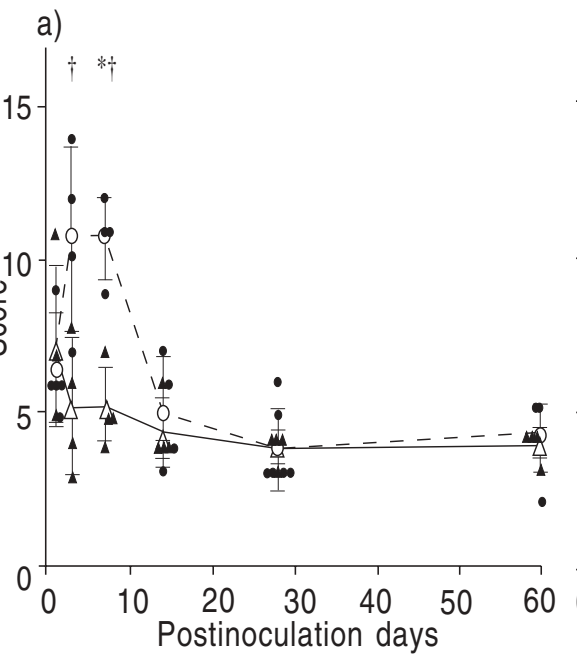

b)

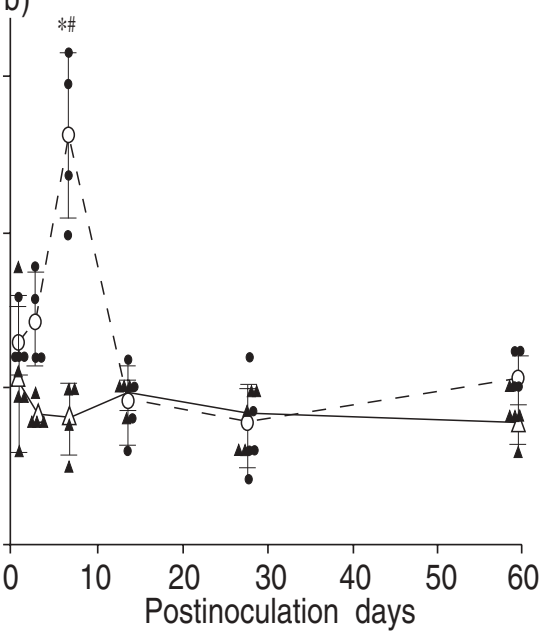

c)

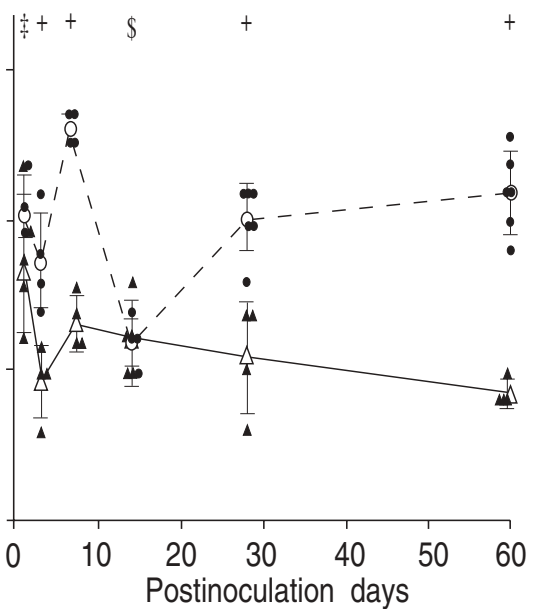

Fig. 7. - Histological score for: a) bronchiolar epithelial necrosis; b) mononuclear cell infiltrates; and c) polymorphonuclear infiltrates, for respiratory syncytial virus (RSV)-inoculated $(\bullet)$ and control guinea-pigs $(\mathbf{\Delta})$ over 60 days. Larger open symbols with bars represent mean \pm SD. a) $\dagger$ : $\mathrm{p}<0.002$, for RSV-inoculated animals $v s$ other days; *: $\mathrm{p}<0.001$, for RSV-inoculated animals, vs control animals; \#: $\mathrm{p}<0.001$ for RSV-inoculated animals, $v s$ other days; $\stackrel{+}{+}<0.02$, for control animals, $v s$ other days; ${ }^{+}: \mathrm{p}<0.009$, for RSV-inoculated $v s$ control animals; $\$$ : $\mathrm{p}<0.003$, for RSVinoculated animals vs other days.

\section{Discussion}

The present study clearly demonstrates that human RSV productively replicates in the guinea-pig lung during acute infection, and can persist as replicating, infectious virus for at least 60 days following experimental, intranasal inoculation of anaesthetized, juvenile guineapigs. This study also demonstrates that experimental RSV infection results in the production of RSV-specific IgG1 antibody, production of bronchiolar inflammation that resembles human acute bronchiolitis, and sequelae of chronic airway inflammation characterized by increased bronchiolar PMN infiltrates.

In this study, the use of immunocytochemistry to ascertain the nature of syncytia produced by RSV provided more sensitivity and specificity for viral quantification, particularly for the detection of small syncytia that could be masked by overgrowth of HEp-2 cells and may not be visualized by conventional detection procedures. It must be noted that the amounts of intrapulmonary, replicating virus reported in this study might be underestimated, since the tissue homogenization procedure may not allow for complete release of intracellularly-associated virus.

Persistent viral infection of the lung has previously been documented in cotton rats treated with immunosuppressive therapy [14], and may also occur in naturally or medically-immunocompromised patients [21], in whom suppression of the inflammatory responses impairs the clearance of virus. However, to our knowledge, this is the first report to describe persistence of intrapulmonary, replicating RSV in an immunocompetent animal model. Thus, the persisting RSV antigen and genome previously shown in guinea-pig after the resolution of acute bronchiolitis [17], corresponds to infectious virus.

There is abundant evidence that acute viral infections can stimulate increases in airway responsiveness in asthmatic patients and in normal subjects, that are related to effects of viral-induced lysis of respiratory epithelial cells and airway inflammation (reviewed in [22]). However a recent study, investigating the effect of RSV on histamine-induced bronchial reactivity in guinea-pigs, demonstrated that airway hyperresponsiveness can persist for at least 6 weeks following infection with human RSV [23]. Accordingly, the persistent, infectious RSV particles demonstrated in this study may represent a potential reservoir of pathogenic antigens, which may stimulate persistent airway hyperresponsiveness. Our findings that RSV-inoculated guinea-pigs had significantly increaed bronchiolar PMN infiltrates, during persistent infection on Days 28 and 60, suggest that chronic airway inflammation may be involved in the pathogenesis of persistent airway hyperresponsiveness after acute bronchiolitis. Further studies are required to test this intriguing possibility.

The results of histological scoring extend our previous observations in this model $[16,17]$ in several aspects. Firstly, the scoring system can be used to distinguish effects of RSV on bronchiolar inflammation before the peak of acute bronchiolitis on Day 7. Secondly, the results of histological scoring show differences in the time course of bronchiolar mononuclear and PMN cell infiltrates over 60 days. Increases in mononuclear infiltrates were observed only during acute bronchiolitis, consistent with previous reports [11-13]. In contrast, bronchiolar PMN infiltrates showed a biphasic pattern, in which significant increases were observed in the RSVinoculated group during acute bronchiolitis and subsequently during persistent infection. These findings demonstrate the importance of extending studies of RSV infection of experimental animals beyond the convalescent phase (10-14 days postinoculation), and further studies are required to elucidate the mechanisms of the second, prolonged increase in bronchiolar PMN infiltrates that we observed in virus-infected guinea-pigs. Finally, the results of histological scoring reveal that sham inoculation produces a transient increase in bronchiolar PMN infiltrates, with resolution by Day 3. These results indicate that the differences in histological scores observed between RSV-infected and control guinea-pigs, from Day 3 onward, are attributable to effects of the virus.

Of particular interest in our study was the demonstration of elevated anti-RSV IgG1 antibody titres in the 
sera of RSV-infected guinea-pigs. It is well-recognized that guinea-pig IgG1 plays a predominant role in the allergic responses to aerosolized antigens, which result in the release of histamine and inflammatory media- tors, such as leukotrienes $[18,24]$. Human studies have provided evidence that elevated levels of RSV-specific IgE and histamine can be detected in nasopharyngeal secretions from children with acute RSV bronchiolitis [8, 9]. Other studies have also demonstrated RSV-specific IgE and tryptase in bronchial lavage recovered from young children with acute bronchiolitis [10], and leukotrienes have been measured in the respiratory tract of children with RSV infection [25]. Thus, the present finding of an RSV-specific IgG1 antibody response in the guinea-pig model of acute bronchiolitis adds support to the notion that RSV itself might act as an allergen and, through an interaction with specific IgE and mast cells or basophils, stimulate the release of histamine and mediators implicated in the pathogenesis of airway hyperresponsiveness.

In summary, the results of this study suggest that the chronic persistence of infectious respiratory syncytial virus in the lung, with the development of respiratory syncytial virus-specific immunoglobulin E responses and chronic airway inflammation, may be important factors in the pathogenesis of postbronchiolitis wheezing and asthma in children. Based on the present observations, studies are now required to determine whether respiratory syncytial virus can cause persistent lung infection in immunocompetent children.

Acknowledgements: The authors thank L. Verburgt for statistical analysis, and S. Greene for excellent photographic assistance.

\section{References}

1. Stott EJ, Taylor G. Respiratory syncytial virus: brief review. Arch Virol 1985; 84: 1-52.

2. McIntosh K, Chanock RM. Respiratory syncytial virus. In: Virology. 2nd edn. Field BN, Knipe DM, et al., eds. New York, Raven Press Ltd, 1990; pp. 1045-1072.

3. Anderson LJ, Parker RA, Strikas RL. Association between respiratory syncytial virus outbreaks and lower respiratory tract deaths of infants and young children. $J$ Infect Dis 1990; 161: 640-646.

4. Pullan CR, Hey EN. Wheezing, asthma, and pulmonary dysfunction 10 years after infection with respiratory syncytial virus in infancy. $B r$ Med $J$ 1982; 284: 16651669.

5. Hall CB, Hall WJ, Gala CL, MaGill FB, Leddy JP. Longterm prospective study in children after respiratory syncytial virus infection. J Pediatr 1984; 105: 358-364.

6. Sly PD, Hibbert ME. Childhood asthma following hospitalization with acute viral bronchiolitis in infancy. Pediatr Pulmonol 1989; 7: 153-158.

7. Sigurs N, Bjarnason R, Sigurbergsson F, Kjellman B, Björkstén B. Asthma and immunoglobulin E antibodies after respiratory syncytial virus bronchiolitis: a prospective cohort study with matched controls. Pediatr 1995; 95: 500-505.

8. Welliver RC, Wong DT, Sun M, Middleton E, Vaughn RS, Ogra PL. The development of respiratory syncytial virus-specific $\operatorname{IgE}$ and the release of histamine in nasopharyngeal secretions after infection. $N$ Engl J Med 1981; 305: 841-846.

9. Welliver RC, Sun M, Rinaldo D, Ogra PL. Predictive value of respiratory syncytial virus-specific IgE responses for recurrent wheezing following bronchiolitis. J Pediatr 1986; 109: 776-780.

10. Everard ML, Fox G, Walls AF, et al. Tryptase and IgE concentrations in the respiratory tract of infants with acute bronchiolitis. Arch Dis Child 1995; 72: 64-69.

11. Hambling MH. Antibody response in guinea-pigs following intranasal inoculation of respiratory syncytial virus. J Path Bact 1966; 91: 625-629.

12. Prince GA, Jenson AB, Horswood RL, Camargo E, Chanock $\mathrm{RM}$. The pathogenesis of respiratory syncytial virus infection in cotton rats. Am J Pathol 1978; 93: 771-792.

13. Graham BS, Perkins MD, Wright PF, Karzon DT. Primary respiratory syncytial virus infection in mice. $J$ Med Virol 1988; 26: 153-162.

14. Johnson RA, Prince GA, Suffin SC, Horswood RL, Chanock RM. Respiratory syncytial virus infection in cyclophosphamide-treated cotton rats. Infect Immun 1982; 37: 369-373.

15. Kimpen JLL, Rich GA, Moha CK, Ogra PL. Mucosal T-cell distribution during infection with respiratory syncytial virus. J Med Virol 1992; 36: 172-179.

16. Hegele RG, Robinson PJ, Gonzales S, Hogg JC. Production of acute bronchiolitis in guinea-pigs by human respiratory syncytial virus. Eur Respir J 1993; 6: 13241331.

17. Hegele RG, Hayashi S, Bramley AM, Hogg JC. Persistence of respiratory syncytial virus genome and protein after acute bronchiolitis in guinea-pigs. Chest 1994; 105: $1848-1854$.

18. Al-laith M, Weyer A, Havet N, Dumarey C, Vargaftig BB, Bachelet M. Immunoglobulin-G-dependent stimulation of guinea-pig lung mast cells and macrophages. Allergy 1993; 48: 608-614.

19. Alwan WH, Record FM, Openshaw PJM. CD4+ T-cells clear virus but augment disease in mice infected with respiratory syncytial virus: comparison with the effects of CD8+ T-cells. Clin Exp Immunol 1992; 88: 527-536.

20. Holland BS, Copenhaver MD. An improved sequentially rejective Bonferroni procedure. Biometrics 1987; 43: 417-423.

21. Hall CB, Powell KR, MacDonald NE, et al. Respiratory syncytial viral infection in children with compromised immune function. $N$ Engl J Med 1986; 315: 77-81.

22. Hegele RG, Hayashi S, Hogg JC, Paré PD. Mechanisms of airway narrowing and hyperresponsiveness in viral respiratory tract infections. Am J Respir Crit Care Med 1995; 151: 1657-1665.

23. Riedel F, Streckert HJ, Oberdieck B, Philippou S, Marek W. Persistence of airway hyperresponsiveness and viral antigen following RSV bronchiolitis in the guinea pig. Eur Respir J 1995; 8 (Suppl. 19): 45S.

24. Watson JW, Conklyn M, Showell HJ. IgG1-mediated acute pulmonary hypersensitivity response in the guineapig: involvement of specific lipid mediators. Am J Respir Crit Care Med 1990; 142: 1093-1098.

25. Volovitz B, Welliver RC, DeCastro G, Krystofik DA, Ogra PL. The release of leukotrienes in the respiratory tract during infection with respiratory syncytial virus: role in obstructive airway disease. Pediatr Res 1988; 24: 504-507. 